\title{
Numerical Study of Magnetoaerodynamic Flow Around a Hemisphere
}

\author{
Nicholas J. Bisek** and Iain D. Boyd‡ \\ University of Michigan, Ann Arbor, Michigan 48109 \\ and \\ Jonathan Poggie \\ U.S. Air Force Research Laboratory, Wright-Patterson Air Force Base, Ohio 45433-7512
}

DOI: $\underline{10.2514 / 1.49278}$

\begin{abstract}
Newly developed computational tools are used to compute hypersonic flow around a hemisphere cylinder that uses a magnet located within the body as a means of heat flux mitigation. These tools include an improved electrical conductivity model and a parallelized three-dimensional magnetohydrodynamic module that is loosely coupled to a three-dimensional fluid code. Several electrical conductivity models are explored for a range of magnetic field strengths. Results show the shock standoff distance increases when the magnetic field is applied, but the distance is highly dependent on the conductivity model selected. The increase in shock standoff distance reduces the gradients in the shock layer, thereby reducing the peak heat flux to the body. However, the total heat flux slightly increases due to additional heating to the aft section of the geometry.
\end{abstract}

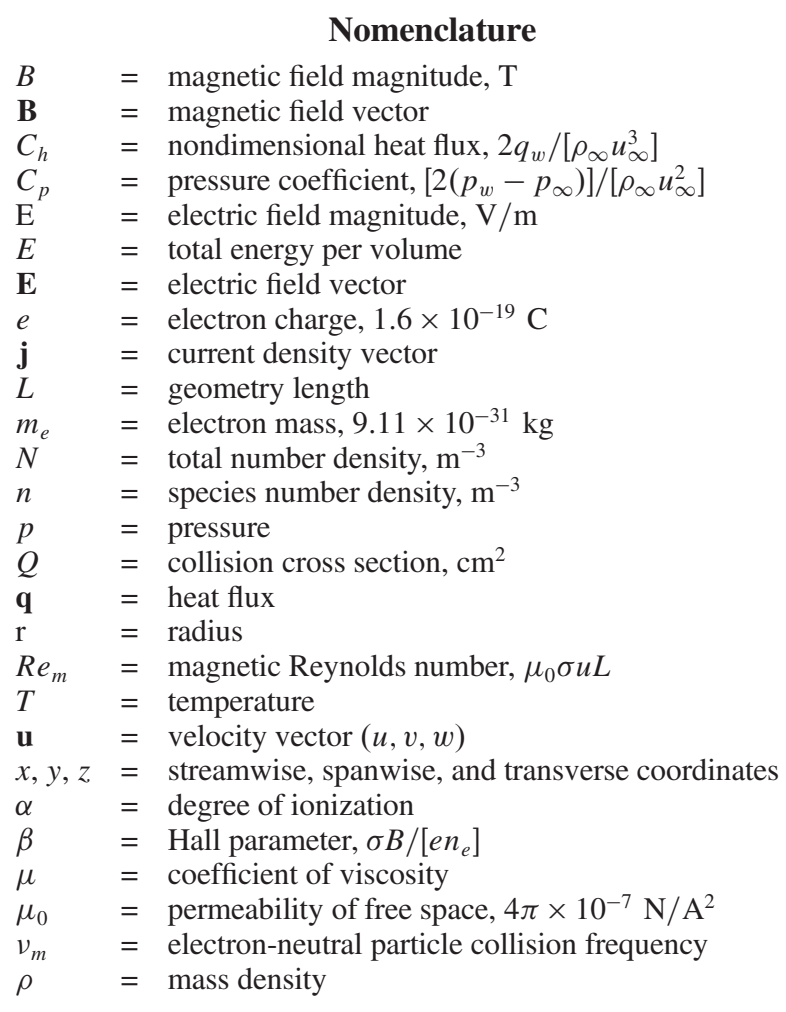

Presented as Paper 2010-0227 at the 48th AIAA Aerospace Sciences Meeting including the New Horizons Forum and Aerospace Exposition, Orlando, FL, 4-7 January 2010; received 8 February 2010; revision received 27 May 2010; accepted for publication 4 June 2010. This material is declared a work of the U.S. Government and is not subject to copyright protection in the United States. Copies of this paper may be made for personal or internal use, on condition that the copier pay the $\$ 10.00$ per-copy fee to the Copyright Clearance Center, Inc., 222 Rosewood Drive, Danvers, MA 01923; include the code 0022-4650/10 and $\$ 10.00$ in correspondence with the CCC.

${ }^{*}$ Graduate Student, Department of Aerospace Engineering; currently at Ohio Aerospace Institute, Wright-Patterson Air Force Base, Ohio. Member AIAA.

'Professor, Department of Aerospace Engineering, 1320 Beal Avenue. Associate Fellow AIAA.

Fenior Aerospace Engineer, AFRL/RBAC, Building 146, Room 215, 2210 Eighth Street. Associate Fellow AIAA.

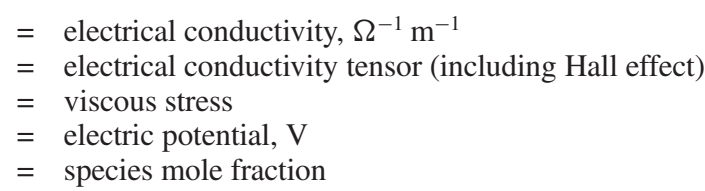

Subscripts

$\begin{array}{ll}\mathrm{n} & =\text { nose } \\ s & =\text { species } \\ \mathrm{w} & =\text { wall } \\ \infty & =\text { freestream }\end{array}$

\section{Introduction}

$\mathbf{T}$ HE idea of using an applied magnetic field to reduce the heat transfer to a hypersonic vehicle has been a topic of scientific research since the late 1950s, when Kantrowitz [1] and Resler and Sears [2,3] conducted the first calculations demonstrating the potential benefits an applied magnetic field has on a weakly ionized flow, a condition typically observed during reentry. The magnetic field, if properly aligned, creates a magnetic force that opposes the incoming flow, effectively increasing the shock standoff distance. The thickening of the shock layer reduces the gradients near the stagnation point and thus lowers the peak heat transfer rate. In the midst of the space race, this novel idea attracted a lot of attention as many groups looked to further explore and refine the semianalytical calculations by making various approximations to the conservation equations. Of these efforts, the work by Bush $[\underline{4}, \underline{5}]$ is considered to be one of the most complete approximate analytic solutions [6]. Bush's approach used a local solution at the stagnation point of the hypersonic flow over an axisymmetric blunt body and predicted significant flow deceleration with the presence of an opposing magnetic field. The first modern computational fluid dynamics (CFD) simulations of the magnetohydrodynamic (MHD) blunt body problem were completed about a decade later by Coakley and Porter [7]. Because of the lack of computational resources at the time, the simulations still required significant simplifications, including the assumptions that the gas was ideal, nonreacting, and inviscid.

The first experimental work to complement the analytical/ computational activity was completed by Ziemer [8] and focused on measuring the shock standoff distance. Bush's approximate results were in reasonable agreement with this experiment. The first heat transfer measurements for this concept were collected in the 
experimental work by Wilkinson [9] for Mach 3 ionized argon at the stagnation point of a blunt cone.

Another experimental effort was conducted by Kranc et al. [10] in the late 1960s. This work provided additional experimental validation sets for the continuing computational efforts, as it explored shock standoff distance and drag measurements for hypersonic flow over two different axisymmetric geometries. These experiments were run in a flow regime where both the viscosity and Hall effect are important, and they confirmed the increase in the shock standoff distance and total drag on the geometry in the presence of an opposing magnetic field. Analysis of the experiment by Nowak et al. [11] and Nowak and Yuen [12] showed the geometries also exhibited an increase in total heating that was attributed to the Hall effect. This was an unanticipated result, because the thickening of the shock layer reduces gradients within the stagnation region, which should reduce the heat flux to the body. In addition, previous semianalytic work had predicted that the Hall effect would only reduce the effectiveness of the magnetic force on increasing the shock standoff distance and total drag on the geometry [13]. Regardless, it was determined that the large magnetic field strength needed to make the technology practical required a magnet that was too heavy to be placed on reentry vehicles, and the research area faded [14].

While hypersonic research continued to experience strong support through the rest of the 20th century due, in part, to various programs like Apollo and shuttle [15], it was not until the mid-1990s that interest in plasma-assisted hypersonic flow control started to reappear [16-18]. This resurgence has been credited to many factors, including the increasing demand for sustained hypersonic flight, rapid access to space, and numerous mechanical and material advances in the area of flight-weight MHD technologies. One of the first to reevaluate the technology using modern CFD techniques was Palmer [19], who performed first-order spatially accurate simulations of the time-dependent Maxwell's equations coupled to the Navier-Stokes equations to analyze a Mars return vehicle. The rising costs for hypersonic experiments and the need for results within a greater range of flowfield conditions for increasing geometric complexity has continued to motivate the development of computational tools that are capable of accurately computing these plasmabased hypersonic flow control devices. This need has spurred numerous computational studies in the recent years, exploring all aspects of plasma-based flow enhancements, including flow control [20-25], local heat load mitigation [26-30], communications blackout [31,32], and MHD power extraction [33-35].

Despite the large financial costs, limited facilities, and technical challenges, some recent experimental studies have been performed by Bityurin et al. [36], Takizawa et al. [37], Kimmel et al. [38], Matsuda et al. [39], and Gülhan et al. [40] to explore electromagnetic effects on hypersonic flows. While these efforts have provided new insight into electromagnetic phenomena in hypersonic flows, more precise measurements, and additional validation exercises for testing the accuracy of fluid-MHD codes, the rising costs (increased maintenance for aging facilities and additional safety protocol) associated with conducting hypersonic experiments greatly limits the number of experiments being conducted. At the same time, supercomputing systems continue to experience exponential performance increases with substantial decreases in cost. This has led to a continued increase in computational research. In fact, Padilla estimates that, if current trends continue, over $70 \%$ of hypersonic research will involve computational analysis by 2020 [15].

This work focuses on the previously mentioned experiment conducted by Kranc et al. [10], which was similar to the semianalytical research conducted by Bush [4,5]. Bush's computational work was previously explored computationally by Gaitonde and Poggie [41,43] and Gaitonde [42]. In the work by Poggie and Gaitonde, several of Bush's [4,5] simplifications were removed, and the Hall effect was added and investigated, while Damevin and Hoffmann [44] explored chemistry effects for a single-temperature model. In both efforts, a simplified model was used to estimate the flow's electrical conductivity. The present work extends these efforts by investigating several electrical conductivity models, including a surrogate model of solutions to Boltzmann's equation. The results show the change in shock standoff distance due to the presence of the magnetic field corresponds very well with the experimental measurements, especially when employing the newly developed surrogate electrical conductivity model. In addition, the solutions show an increase in total heating to the geometry, which is consistent with the observations made by Nowak et al. [11] and Nowak and Yuen [12]. The increase in total heating is due to a slight increase in heating on the cylinder section (aft) of the geometry.

\section{Method}

\section{A. Governing Equations}

Flowfield results are obtained using CFD to solve the NavierStokes equations. The computations are executed using the Michigan aerothermodynamic Navier-Stokes (LEMANS) code, which was developed at the University of Michigan $[45,46]$.

LEMANS is a general two-dimensional (2-D)/axisymmetric/ three-dimensional (3-D), parallel, unstructured finite-volume CFD code. The numerical fluxes between cells are discretized using a modified Steger-Warming flux vector splitting (FVS) scheme, except near shock waves. In these regions, the original StegerWarming FVS scheme is used, because it provides sufficient dissipation to accommodate the discontinuity [47]. LEMANS is able to employ a two-temperature or three-temperature model to account for thermal nonequilibrium and a standard finite-rate chemistry model for nonequilibrium chemistry. The two-temperature model assumes a single temperature $T$ and accounts for the translational and rotational energy modes of all species, while the vibrational energy mode is accounted for by a separate temperature $T_{v e}$. In the threetemperature model, the rotational energy mode is independent of the translational energy mode [48].

For a single temperature (local thermodynamic equilibrium) model with MHD, but without finite-rate chemistry, the conservation equations are

$$
\begin{gathered}
\frac{\partial \rho_{s}}{\partial t}+\nabla \cdot\left(\rho_{s} \mathbf{u}\right)=0 \\
\frac{\partial \rho \mathbf{u}}{\partial t}+\nabla \cdot(\rho \mathbf{u} \mathbf{u}+p \mathbf{I}-\tau)=\mathbf{j} \times \mathbf{B} \\
\frac{\partial E}{\partial t}+\nabla \cdot[(E+p) \mathbf{u}-\tau \cdot \mathbf{u}+\mathbf{q}]=\mathbf{j} \cdot \mathbf{E}
\end{gathered}
$$

where $\mathbf{u u}$ in the conservation of momentum equation is the $3 \times 3$ tensor containing all the products of the components of the velocity vector:

$$
\mathbf{u} \mathbf{u}=\left[\begin{array}{ccc}
u^{2} & u v & u w \\
v u & v^{2} & v w \\
w u & w y & w^{2}
\end{array}\right]
$$

LEMANS assumes the fluid is continuous and Newtonian. It also assumes Stokes' hypothesis when determining the viscous stresses:

$$
\tau_{i j}=\mu\left(\frac{\partial u_{j}}{\partial x_{i}}+\frac{\partial u_{i}}{\partial x_{j}}\right)-\frac{2}{3} \mu \nabla \cdot \mathbf{u} \delta_{i j}
$$

The total energy deposition term $\mathbf{j} \cdot \mathbf{E}$ appears on the right side of Eq. (3). The conservation of momentum equation is modified to include a magnetic force $\mathbf{j} \times \mathbf{B}$ on the right-hand side of Eq. (2). These additions constitute the effects the current density $\mathbf{j}$, the electric field $\mathbf{E}$, and the magnetic field $\mathbf{B}$ have on the flow.

\section{B. Low-Magnetic Reynolds Number Approximation}

The three additional variables appearing in the governing equations ( $\mathbf{j}, \mathbf{B}$, and $\mathbf{E}$ ) are determined by first noting that the magnetic Reynolds number [Eq. (5)] is small for the cases of interest:

$$
R e_{m}=\mu_{0} \sigma u L
$$


where the permeability of free space $\mu_{0}=4 \pi \times 10^{-7} \mathrm{~N} / \mathrm{A}^{2}, \sigma$ is the electrical conductivity, $u$ is the streamwise velocity, and $L$ is the reference length. Consequently, it can be shown that the induced magnetic field can be neglected [49]. This means only external magnetic fields are present in the flow (and must be specified). The current density and electric fields are determined by solving the current continuity equation, which has the form of a Poisson equation, as seen in Eq. (6):

$$
\nabla \cdot \tilde{\sigma} \cdot[-\nabla \phi+\mathbf{u} \times \mathbf{B}]=0
$$

where $\tilde{\sigma}$ is the electrical conductivity tensor, a compact way of accounting for the Hall effect [50] that is described in the next section. The electric potential $\phi$ is computed using a finite-volume method and appropriate boundary conditions, as outlined in previous work [51]. The electric field is computed directly from the electric potential $(\mathbf{E}=-\nabla \phi)$, which allows the electric current $\mathbf{j}$ to be computed using a generalized form of Ohm's law $(\mathbf{j}=\tilde{\sigma} \cdot[\mathbf{E}+$ $\mathbf{u} \times \mathbf{B}])$. Full details and validation of the MHD solver are available in [51], while [52] provides details on its parallelization and implementation into the fluid code.

\section{Hall Effect}

As seen in Eq. (6), the MHD module incorporates the tensor nature of the electrical conductivity by following the computational work of Gaitonde [42] and Gaitonde and Poggie [50]. This approach provides a compact way of accounting for ion slip and the Hall effect, phenomena that can change the components of the magnetic force and joule heating of the fluid if the parameters are sufficiently large. Equation (7) shows the electrical conductivity tensor with the Hall effect in Cartesian coordinates:

$$
\tilde{\sigma}=\frac{\sigma}{B^{2}\left(1+\beta^{2}\right)}\left[\begin{array}{ccc}
B^{2}+\beta^{2} B_{x}^{2} & \beta\left(\beta B_{x} B_{y}-B B_{z}\right) & \beta\left(\beta B_{x} B_{z}+B B_{y}\right) \\
\beta\left(\beta B_{y} B_{x}+B B_{z}\right) & B^{2}+\beta^{2} B_{y}^{2} & \beta\left(\beta B_{y} B_{z}-B B_{x}\right) \\
\beta\left(\beta B_{z} B_{x}-B B_{y}\right) & \beta\left(\beta B_{z} B_{y}+B B_{x}\right) & B^{2}+\beta^{2} B_{z}^{2}
\end{array}\right]
$$

where $\sigma$ is the electrical conductivity of the fluid. $B_{x}, B_{y}$, and $B_{z}$ are the components of the magnetic field vector, and $B$ is its magnitude. The Hall parameter $\beta$ is defined in Eq. ( $\underline{8})$ :

$$
\beta=\frac{\sigma B}{e n_{e}}
$$

where $\sigma$ is the electrical conductivity, an elemental charge $e=1.6022 \times 10^{-19} \mathrm{C}$, and $n_{e}$ is the electron number density.

Validation of the Hall effect is performed by using a computational study developed by Hurwitz et al. [53] and rigorously explored by Oliver and Mitchner [54]. In the experiment, finite segmented

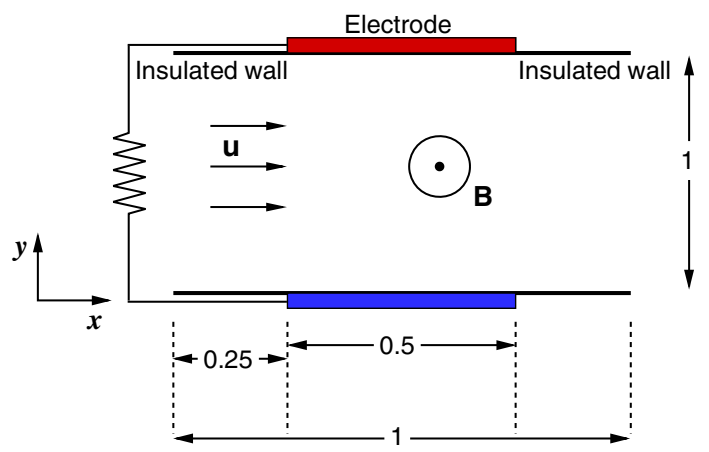

Fig. 1 Schematic of the channel flow with finitely segmented electrodes. Units are in meters. electrodes are infinitely repeated along the two walls of a channel, as seen in Fig. 1. An externally applied magnetic field is positioned perpendicular to the channel velocity $\mathbf{u}$.

This exercise is inherently 2-D, but it is transformed into three dimensions by allowing the channel walls to be infinitely tall. This modification was necessary, since the MHD module is currently only suited for 3-D simulations. Symmetric boundary conditions are applied to the top and bottom planes of the domain, so the actual height of the channel domain is set to a finite value of $0.1 \mathrm{~m}$ for the simulation.

Because the channel is infinitely long, periodic boundary conditions are developed and employed at the domain inlet and outlet. Oliver and Mitchner demonstrated that two of the four global conditions (i.e., streamwise and spanwise current or voltage) are required to determine a unique solution [54]. For this analysis, the applied voltage between the electrode pairs and neighbors is specified (streamwise and spanwise voltages). The voltage between an electrode pair (i.e., the spanwise voltage) is set to unity, while the voltage between electrode neighbors (along the same wall) is set, based on specified scenario. The potential along the insulated wall is determined by setting the current into the insulator to zero $(\mathbf{j} \cdot \mathbf{n}=0)$.

A two-point overlapping stencil, shown in Fig. 2, transfers information between the periodic inlet and outlet planes, while either adding or subtracting the specified streamwise voltage $\left(\Delta \phi_{x}\right)$. Since the interior cells are computed using a second-order method, the twopoint stencil provides sufficient information to allow the last interior points to be accurately updated. A row of cells starts at the inlet and ends at the outlet (constant $y$ and $z$ ). The inlet ghost cell is set equal to the last interior cell next to the outlet (minus the applied streamwise voltage). Likewise, the outlet's ghost cell is set equal to the first interior cell next to the inlet (plus the applied streamwise voltage).

Oliver and Mitchner [54] formulated this problem so that the fluid velocity field did not affect the solution as long as $\nabla \times(\mathbf{u} \times \mathbf{B})=0$. During one iteration of the flow solver, the MHD routine is executed, assuming the velocity profile is only a function of the distance between the channel walls, $\mathbf{u}=f(y)$. This assumption satisfies $\nabla \times(\mathbf{u} \times \mathbf{B})=0$ as long as $\mathbf{B}=f(z)$. The velocity profile is assumed to be a fully developed Poiseuille flow between parallel plates [도], as seen in Eq. ()):

$$
\mathbf{u}=f(y)=u_{\max }\left(1-\frac{\left(y-y_{h}\right)^{2}}{h^{2}}\right)
$$

where $u_{\max }$ is the maximum velocity and is set to unity for this scenario $\left(u_{\max }=1 \mathrm{~m} / \mathrm{s}\right)$. The $y$ location is measured from the center of the channel width $\left(y_{h}=0.5 \mathrm{~m}\right)$, so $h=0.5 \mathrm{~m}$ is the channel halfwidth.

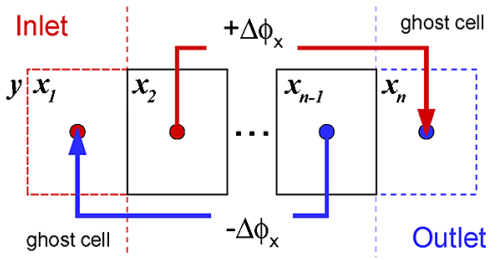

Fig. 2 Cartoon of a two-point stencil used for period boundary conditions. 


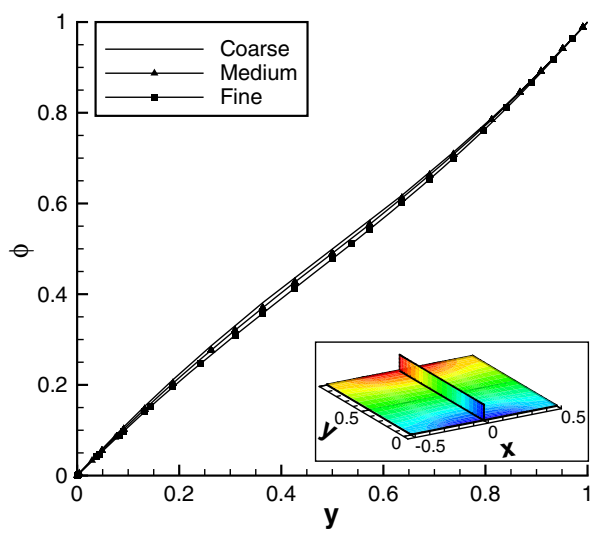

a) $\boldsymbol{x}=\mathbf{0} \mathrm{m}$

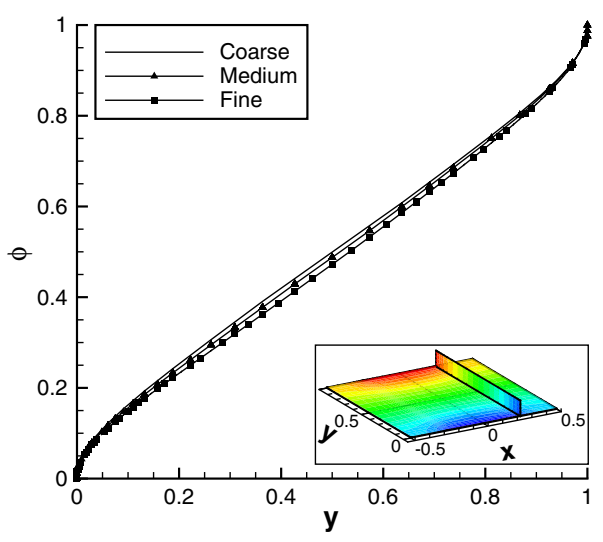

b) $x=0.25 \mathrm{~m}$

Fig. 3 Electric potential $\phi$ between two segmented electrodes at two different locations $(x=0$ and $0.25 \mathrm{~m})$ for various grids $(z=0 \mathrm{~m})$.

A grid convergence study was performed using nonreacting argon gas as the fluid, with a constant electrical conductivity $\sigma=$ $1 \Omega^{-1} \mathrm{~m}^{-1}$. The channel walls are $1 \mathrm{~m}$ apart, and the segmented electrodes are $0.5 \mathrm{~m}$ wide, with $0.5 \mathrm{~m}$ of insulated wall between electrode neighbors so that the simulated domain has a length of $1 \mathrm{~m}$ in both the $x$ and $y$ directions. The channel walls are set to a height of $0.1 \mathrm{~m}$ in the $z$ direction, with symmetric boundaries applied at the $z=0,0.1 \mathrm{~m}$ planes. Periodic boundaries are applied at the inlet and outlet ( $x=-0.5$ and $0.5 \mathrm{~m}$, respectively), with a streamwise potential equal to zero $\left(\Delta \phi_{x}=0\right)$. The spanwise potential is set to $1 \mathrm{~V}$ by setting the bottom electrode $(y=0 \mathrm{~m})$ to zero and the potential at the top electrode $(y=1 \mathrm{~m})$ to $1 \mathrm{~V}$.

The grid uses exponential spacing along the wall surface, such that cell clustering occurs near the junction between the insulated wall and the electrode. Exponential spacing is employed between the two walls, such that cell clustering occurs near each surface. Uniform spacing is employed along the height of the wall ( $z$ direction). The coarse grid employs 50 points along the wall ( $x$ direction), 20 points between the walls ( $y$ direction), and 4 points in the $z$ direction. Two additional, doubly refined grids are also developed: $100 \times 40 \times 8$ (medium) and $200 \times 80 \times 16$ (fine).

Grid independence is assessed by comparing solutions of the electric potential $\phi$ for the scenario without a magnetic field $(\mathbf{B}=0)$. Since the wall is infinitely tall, the solution in the $z$ direction is constant and is only plotted along the $z=0 \mathrm{~m}$ plane. Extracting solutions of $\phi$ at two slices of the domain $(x=0$ and $0.25 \mathrm{~m})$ in Figs. $3 \mathrm{a}$ and $3 \mathrm{~b}$ show the potential does not vary significantly between the medium and fine grids, so the medium solution is considered grid-independent and is employed in the rest of the section.

Without the magnetic field, the Hall effect is nullified, and the electrical conductivity tensor reverts to a scalar. With the streamwise voltage set to zero, the resultant electric potential solution is symmetric about the center of the electrode, as seen in Fig. 4, where Fig. 4a is obtained by Gaitonde [42] and Fig. 4b is obtained using the medium grid. The current lines are also symmetric about the center of the electrode and are primarily created in the $y$ direction, as seen in Fig. 5.

To test the Hall effect, a scenario used by Hurwitz et al. [53] is simulated. In this case, a $1 \mathrm{~T}$ uniform magnetic field is externally applied. The magnetic field is aligned with the $z$ axis, whereas the velocity is aligned with the $x$ axis. The spanwise voltage between an electrode pair is kept at $1 \mathrm{~V}$, but the electrode pair is offset by $0.28 \mathrm{~m}$ for the Hall parameter of one $(\beta=1)$, as seen in Fig. 6. The streamwise voltage is also specified $\left(\Delta \phi_{x}=0.4305 \mathrm{~V}\right)$.

Hurwitz et al. [53] computed the potential and electric field, as seen in Fig. 7a. Current flows along a diagonal of the squares, seen in Figs. $7 \mathrm{a}$ and $\overline{7 b}$ (i.e., the current lines cross the orthogonal squares in the figures) [56]. The Hall effect creates values in the offdiagonal components of the electrical conductivity tensor, seen in Eq. (7). The antisymmetric components of the conductivity tensor result in a stretching of the streamwise component of the current density vector, $j_{x}$.

While the computed solution (Fig. 7b) closely resembles Hurwitz's [53] semianalytic solution, it is not identical. Hurwitz assumes the streamwise current $j_{x}$ is zero when it is far from the wall

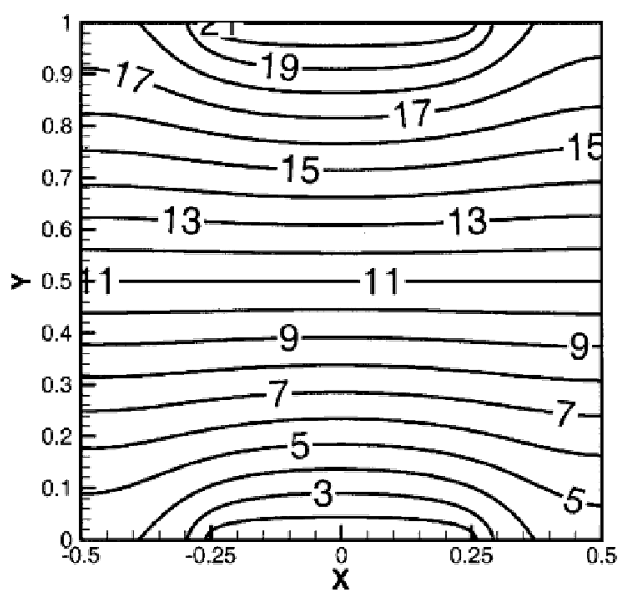

a) From Gaitonde [42]

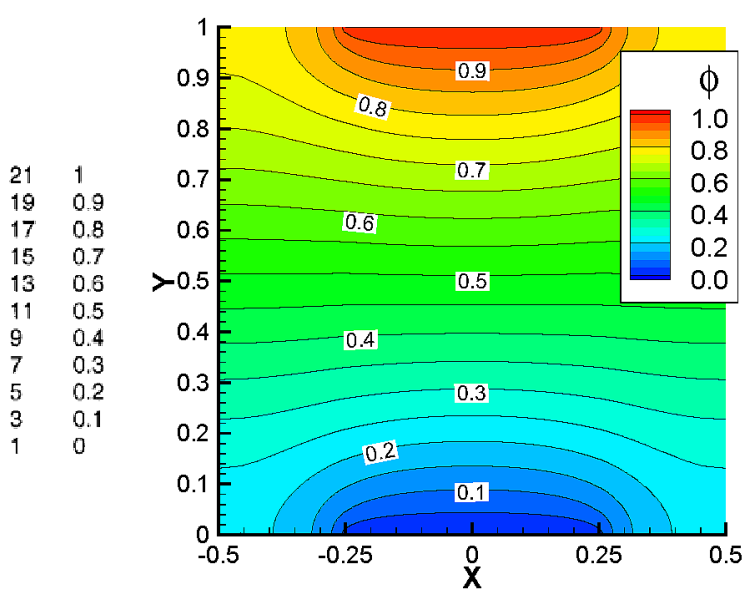

b) Medium grid $(100 \times 40 \times 8)$ at $z=0$

Fig. 4 Electric potential contours for the segmented electrode channel without a magnetic field and constant electrical conductivity $(B=0$, $\sigma=1 \Omega^{-1} \mathrm{~m}^{-1}$, and $\Delta \phi_{x}=0$ ). 


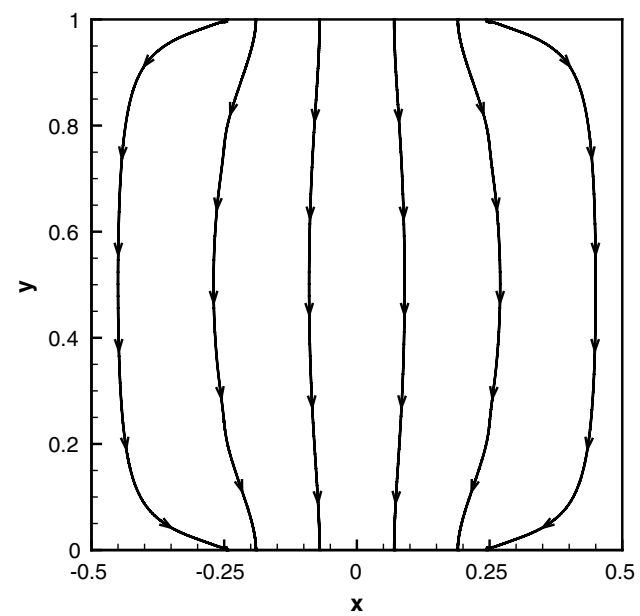

Fig. 5 Current density streamlines between two segmented electrodes without the Hall effect $\left(\mathrm{B}=0, \sigma=1 \Omega^{-1} \mathrm{~m}^{-1}\right.$, and $\left.\Delta \phi_{x}=0\right)$.

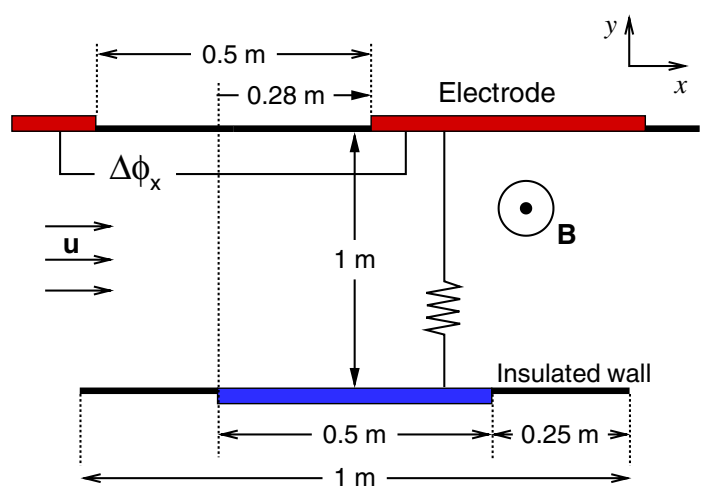

Fig. 6 Schematic of channel flow with finitely segmented electrodes used by Hurwitz et al. [53] with Hall effect $\left(B_{z}=1 \mathrm{~T}, \sigma=1 \Omega^{-1} \mathrm{~m}^{-1}\right.$, $\beta=1$, and $\Delta \phi_{x}=0.4305 \mathrm{~V}$ ).

(i.e., $y=0.5 \mathrm{~m}$ ). While this is a reasonable approximation, it is not completely accurate and is not enforced in the computational results seen in Fig. 7b. Nonetheless, the figures portray similar characteristics and indicate that the Hall effect is successfully implemented.

\section{Electrical Conductivity}

The experiment performed by Kranc et al. [10] used preionized $\operatorname{argon}\left(\mathrm{Ar}, \mathrm{Ar}^{+}\right.$, and e). The electrical conductivity profile for weakly ionized argon is shown in Fig. $\underline{8}$, as adapted from Lin et al. [57]. As seen in the figure, the electrical conductivity exhibits two distinct regions: namely, weakly ionized $(T \lesssim 10,000 \mathrm{~K})$ and fully ionized $(T>10,000 \mathrm{~K})$. Both regions display exponential growth with temperature, which means a highly accurate conductivity model is important in order to accurately capture its behavior across the entire temperature range.

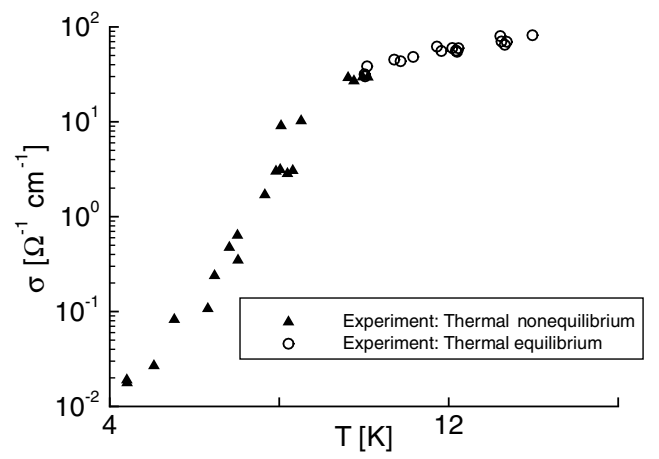

Fig. 8 Electrical conductivity of $\operatorname{argon}(p=0.013 \mathrm{~atm})$, reproduced from Lin et al. [57].

Three different electrical conductivity models were explored for this work. Raizer developed an electrical conductivity model that is an exponential function of temperature, assuming that electronneutral collisions affect the conductivity more than the electron-ion collisions and that the ionization is in thermal equilibrium [58], as seen in Eq. (10):

$$
\sigma=83 \times \mathrm{e}^{-36,000 / \mathrm{T}} \Omega^{-1} \mathrm{~cm}^{-1}
$$

where the temperature $T$ is specified in Kelvins. This model is considered valid for air, nitrogen, and argon at $p=1$ standard atmosphere (atm) for a temperature range of 8000 to $14,000 \mathrm{~K}$. The model's coefficients $(83$ and $-36,000)$ can be adjusted, depending on the temperature range, pressure, or gas composition of interest, but they are used as specified for this study.

Chapman and Cowling developed a model (as described by Cambel [59]) for a weakly ionized gas by assuming a coupling between the charge and mass diffusion terms and assuming that the resultant electron energy distribution function from solutions to Boltzmann's equation is only a function of this coupled, binary diffusion coefficient. This assumption results in a semianalytic model for the electrical conductivity, as seen in Eq. (11):

$$
\sigma=3.34 \times 10^{-10} \frac{\alpha}{Q \sqrt{\mathrm{T}}} \Omega^{-1} \mathrm{~cm}^{-1}
$$

where $Q \mathrm{~cm}^{2}$ is the collision cross section of the gas, and the degree of ionization $\alpha=\Sigma n_{\text {ions }} / N$. One limitation of using the Chapman and Cowling model is that $Q$ must be determined by an outside source (i.e., reference tables, theoretical model, etc.). For this work, the collision cross section is taken to be the total collision cross section for argon-argon collisions using the hard sphere model [60]. The diameter of argon is $4.04 \times 10^{-10} \mathrm{~m}$ [61], so $Q=\pi \overline{d^{2}}=$ $\simeq 5 \times 10^{-15} \mathrm{~cm}^{2}$. This assumption is made, because it is unclear what the best choice for $Q$ should be and because it produces results that are consistent with other models studied in previous work $[51,52]$. Since the model depends on the degree of ionization and temperature, it contains an indirect correlation to pressure and is valid for the cases of interest in this work.

A surrogate model of solutions to Boltzmann's equation is the third electrical conductivity model studied. A second-order

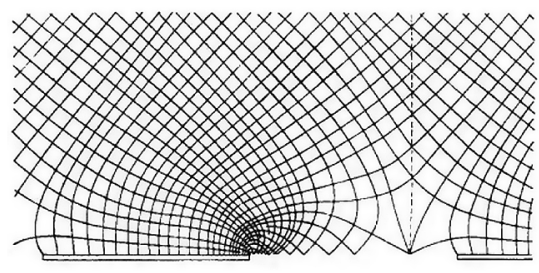

a) Reproduced from Hurwitz et al. [53]

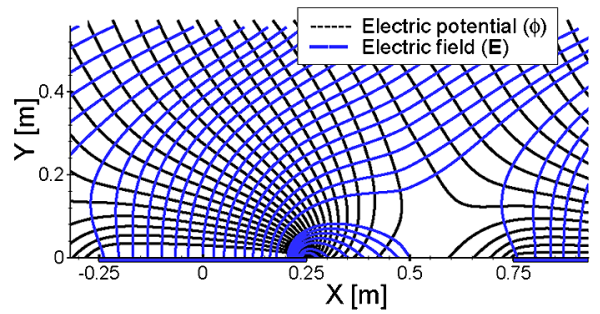

b) Medium grid $(100 \times 40 \times 8)$ at $z=0$

Fig. 7 Potential contours and electric field streamlines between segmented electrodes with the Hall effect $\left(B_{z}=1 \mathrm{~T}, \sigma=1 \Omega^{-1} \mathrm{~m}^{-1}, \beta=1\right.$, and $\left.\Delta \phi_{x}=0.4305 \mathrm{~V}\right)$. 
polynomial response surface (PRS) is employed to capture the behavior of the solutions to Boltzmann's equations by developing a 3-D design of experiment (DOE). Solutions to Boltzmann's equation are determined using a Boltzmann solver developed by Weng and Kushner [62]. The Boltzmann solver's input parameters, namely, $\mathrm{E} / N, \chi_{\mathrm{Ar}}$, and $\chi_{\mathrm{Ar}^{+}}$, define the three dimensions of the DOE ( $\chi_{e}$ is unnecessary because of the assumed local charge neutrality). Since the solutions from the Boltzmann solver rely on the mole fractions, the model automatically incorporates a pressure dependence and is valid as long as the species fractions are within the DOE. General details of this approach were discussed in previous work [52], but specific modifications have been introduced here to improve the accuracy of the model.

Solutions to the learning and testing points required by the DOE are obtained from individual Boltzmann solutions that account for electron-electron collisions. Figure 9 plots the resulting electrical conductivity contours, which show a region of high conductivity for low $\chi_{\mathrm{Ar}}$ (high degree of ionization) and a weak normalized electric field $\mathrm{E} / N$.

The correlation between the electrical conductivity and $\mathrm{E} / N$ is anticipated by factoring the electron number density out of the definition of electrical conductivity for a dc current $\left(\sigma=e^{2} n_{e} /\right.$ $m_{e} v_{m}$ ) to yield $\sigma / n_{e} \sim v_{m}^{-1}$. Since the electron collision frequency $v_{m}$ usually increases with increasing $\mathrm{E} / N$, as seen in Fig. 10, the electrical conductivity should decrease with increasing electric field strength.

An open-source MATLAB ${ }^{\circledR}$ library, SURROGATES Toolbox [63], was used to create the PRS surrogate model. To improve the accuracy of the model, it is useful to transform the function that the PRS is trying to mimic by reducing the range of the dependent variable. Dividing the electrical conductivity by the degree of ionization does not require any additional variables, since $\alpha=$ $\Sigma \chi_{\text {ions }}=\chi_{\mathrm{Ar}^{+}}$, but it does normalize the dependent variable. However, this can lead to a division by zero error when $\alpha=0$, so the formulation of the dependent variable is inverted. This formulation is similar to the Chapman-Cowling model, which also uses the degree of ionization in the numerator. Since this results in a small solution range for the cases of interest $\left(10^{-8} \Omega \cdot \mathrm{m} \leq \alpha / \sigma \leq 10^{-6} \Omega \cdot \mathrm{m}\right)$, the natural logarithm is applied to the dependent variable. Equation (12) lists the model formulation provided to the SURROGATES Toolbox:

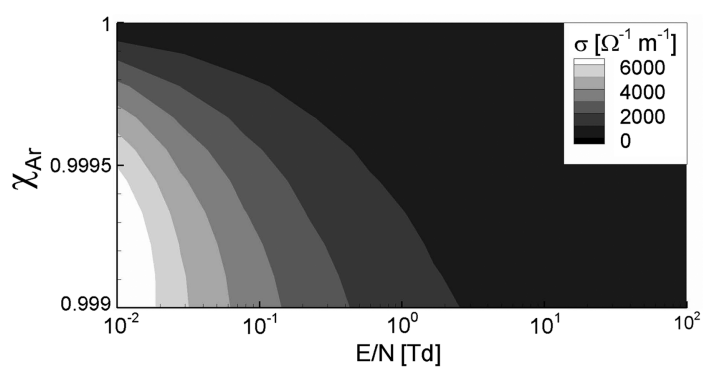

Fig. 9 Electrical conductivity contours for weakly ionized argon.

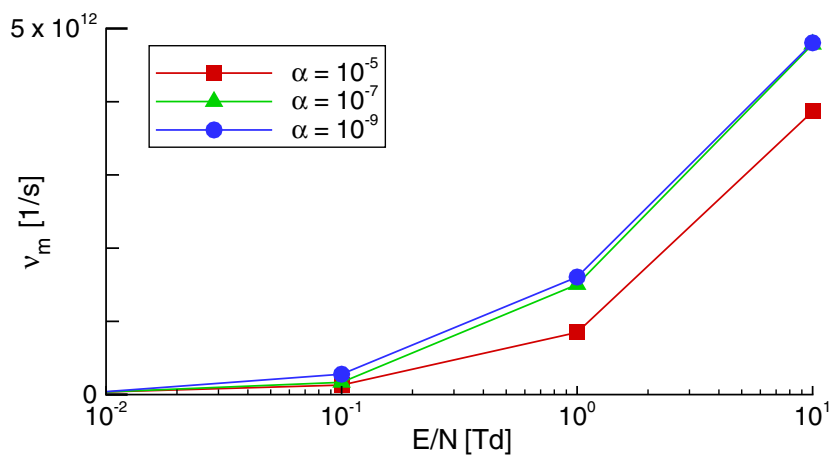

Fig. 10 Electron collision frequency for weakly ionized argon at $p=1$ atm.

$$
\ln \left(\frac{\alpha}{\sigma}\right)=f\left(\mathrm{E} / N, \chi_{\mathrm{Ar}}, \chi_{\mathrm{Ar}^{+}}\right)
$$

Since the model is a function of the natural logarithm and the degree of ionization, the electrical conductivity must be extracted from the model's solution by dividing the degree of ionization by the exponential function of the model's prediction. The resulting electrical conductivity predicted will always be positive $(\sigma \geq 0)$.

Although this formulation of the PRS model incurs additional computational expense (i.e., evaluation of the exponential function and computing $\alpha$ ), higher accuracy is achieved with lower-order PRS models, because the DOE's surface gradients are reduced. This improvement may lead to a reduction in total computing expense for a given scenario, because a lower-order PRS model may be sufficient for the simulations of interest. The model accuracy is determined by computing the mean absolute error (MAE) and the mean absolute percent error (MAPE) from the additional testing points computed using the Boltzmann solver:

$$
\begin{gathered}
\text { MAE }=\frac{1}{n} \sum_{i=1}^{n}|\hat{\sigma}-\sigma|_{i} \\
\text { MAPE }=\frac{1}{n} \sum_{i=1}^{n}\left|\frac{\hat{\sigma}-\sigma}{(\hat{\sigma}+\sigma) / 2}\right|_{i}
\end{gathered}
$$

where $\hat{\sigma}$ is the solution computed by the Boltzmann solver, and $\sigma$ is the solution computed by the model. The percent error is the normalized percent error to remove the bias when evaluating an overprediction [64]. A second-order PRS model is assumed to have sufficient accuracy and acceptable computational cost of the scope of this paper. A summary of the second-order PRS performance metrics yields a MAPE of $16.37 \%$ and a MAE of $90.72 \Omega^{-1} \mathrm{~m}^{-1}$. The second-order PRS model is listed in Eq. (15):

$$
\begin{gathered}
\sigma=\frac{\chi_{e}}{\exp (\mathbf{P R S})} \\
\text { PRS }=-842.64+128.02(\mathrm{E} / N)+2558.28\left(\chi_{\mathrm{Ar}}\right) \\
-4112.52\left(\chi_{\mathrm{Ar}^{+}}\right)-4.82(\mathrm{E} / N)^{2}-118.25(\mathrm{E} / N)\left(\chi_{\mathrm{Ar}}\right) \\
-121.33(\mathrm{E} / N)\left(\chi_{\mathrm{Ar}^{+}}\right)-1732.34\left(\chi_{\mathrm{Ar}}\right)^{2} \\
+3229.51\left(\chi_{\mathrm{Ar}}\right)\left(\chi_{\mathrm{Ar}^{+}}\right)-7342.51\left(\chi_{\mathrm{Ar}^{+}}\right)^{2}
\end{gathered}
$$

where $\mathrm{E} / N$ is normalized from 0 to 1 for a range of 0.01 to 100 townsend $(\mathrm{Td})\left(1 \mathrm{Td}=10^{-17} \mathrm{~V} \cdot \mathrm{cm}^{2}\right)$. The species mole fractions $\chi_{s}$ are used directly in the equation, which was developed for an ionized mole fraction of less than $1 \%$ (i.e., $\chi_{\mathrm{Ar}^{+}}<0.01$ ). However, the model could be expanded to accommodate a large range of mole fractions if necessary. While MAPE may seem relatively high, the result can be misleading, since a large portion of the DOE consists of lower electrical conductivity values, as seen in Fig. 9 (i.e., the MAPE can be quite large when the local solution produced by the Boltzmann solver is small).

\section{E. Viscosity Model}

Chemically nonreacting, thermodynamic equilibrium simulations are computed using the variable hard sphere (VHS) viscosity model. The VHS model is used because the viscosity is assumed to only be a function of temperature, since the species present (argon, argon ion, and electrons) have a single energy mode and are chemically nonreacting:

$$
\mu=\mu_{\text {ref }}\left(\frac{T}{T_{\text {ref }}}\right)^{\omega}
$$

where $\mu$ is the viscosity, the reference viscosity coefficient $\mu_{\text {ref }}=$ $2.117 \times 10^{-5} \mathrm{~N} \mathrm{~s} / \mathrm{m}^{2}$ for a reference temperature $\mathrm{T}_{\text {ref }}=273 \mathrm{~K}$ and a viscosity index $\omega=0.81$. This method, as outlined by Schwartzentruber et al. [65], requires several reference coefficients, which are listed in [60]. 


\section{Results}

Three-dimensional calculations were carried out for Mach 4.75 argon flow over a hemisphere cylinder, which was originally studied experimentally by Kranc et al. [10]. The forebody hemisphere has a radius of 0.75 in. $\left(r_{\mathrm{n}}=0.01905 \mathrm{~m}\right)$, and the geometry is mounted parallel to the freestream, as seen in Fig. 11.

In the experiments, the freestream flow was composed of strongly ionized argon (Kranc et al. [10] estimates the degree of ionization $\alpha=0.025)$, which was produced by a plasma torch (dc arc heater). The heater was located before the converging-diverging nozzle, which accelerated the gas into the test chamber. Kranc et al. [10] state that the electrons were frozen in the nozzle, and that the flow was not chemically reacting after it was initially ionized by the heater. The flow conditions reported by Kranc et al. are listed in Table 1 .

A structured grid was generated using two grid domains. The first domain includes the hemispherical forebody, while the second accommodates the rest of the geometry. While the baseline flow solution (the flow without the magnetic field) is axisymmetric, the rest of the simulations were computed using a 3-D grid, because the MHD routine is currently only implemented for 3-D domains.

The grid was generated with equal spacing along the hemisphere portion of the geometry (first domain) and gradually increases in spacing along the remaining surface (second domain). Grid points were equally spaced around the circumference of the geometry, and the radial points were algebraically spaced to increase the number of points close to the body. As a result, cell clustering occurred primarily in the hemispherical forebody and near the body surface. The baseline grid used 50 points along the body (30 in the hemispherical region), 30 points along one quarter of the circumference, and 30 radial points. Two doubly refined grids were also used in the grid convergence study, giving the following set of computational meshes: $50 \times 30 \times 30$ (coarse), to $100 \times 60 \times 60$ (medium), to $200 \times 120 \times 120$ (fine).

Figure 12 plots the pressure coefficient and nondimensional heat flux for the baseline flow along the surface of the geometry, as defined in Eqs. (17) and (18), respectively:

$$
\begin{gathered}
C_{p}=\frac{p_{w}-p_{\infty}}{1 / 2 \rho_{\infty} u_{\infty}^{2}} \\
C_{h}=\frac{q_{w}}{1 / 2 \rho_{\infty} u_{\infty}^{3}}
\end{gathered}
$$

where $q_{w}$ is the total heat flux to the wall. The grid convergence study showed little difference between the medium and fine grids; therefore, the medium grid was considered sufficiently refined and was used for the rest of the analysis.

A closer examination of the freestream conditions (specifically, the degree of ionization), reveals Kranc et al. [10] estimated $\alpha$ using tables from Arave and Huseley [66]. While this approach may be approximately correct, the degree of ionization is better estimated by using the Saha equation for a singly ionized atomic gas [67]:

$$
\frac{\alpha^{2}}{1-\alpha^{2}} p=3.16 \times 10^{-7} \mathrm{~T}^{5 / 2} \exp \left(-\frac{\varepsilon_{i}}{k \mathrm{~T}}\right)
$$

where $p$ is the pressure in atmospheres, $T$ is the temperature in Kelvin, Boltzmann's constant $k=1.3807 \times 10^{-23} \mathrm{~J} / \mathrm{K}$, and $\varepsilon_{i}$ is the

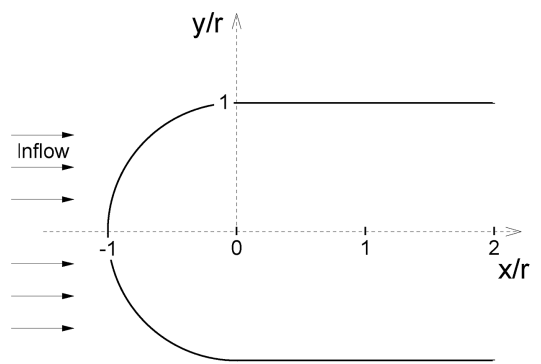

Fig. 11 Hemisphere-capped geometry (adapted from [10]).
Table 1 Flow conditions for the MHDheat-shield experiment as reported by Kranc et al. [10]

\begin{tabular}{lc}
\hline \hline Parameter & Value \\
\hline$M$ & 4.75 \\
$u_{\infty}$ & $3000.0 \mathrm{~m} / \mathrm{s}$ \\
$\mathrm{T}_{\infty}$ & $1100.0 \mathrm{~K}$ \\
$\mathrm{~T}_{\mathrm{w}}$ & $300.0 \mathrm{~K}$ \\
$\mathrm{p}_{\infty}$ & $27.8 \mathrm{~Pa}$ \\
$\rho_{\infty}$ & $1.035 \times 10^{-4} \mathrm{~kg} / \mathrm{m}^{3}$ \\
$n_{\mathrm{e}}$ & $4 \times 10^{19} \mathrm{~m}^{-3}$ \\
$\alpha$ & 0.025 \\
$\mathrm{r}_{\mathrm{n}}$ & $0.01905 \mathrm{~m}$ \\
$\mu_{\infty}$ & $8 \times 10^{-5} \mathrm{~kg} / \mathrm{m} \cdot \mathrm{s}$ \\
$R e_{L}$ & $3880 \mathrm{~m}^{-1}$ \\
$R e$ & 74 \\
\hline \hline
\end{tabular}

ionization energy required to remove the electron from the atom in the gas considered. The ionization potential for argon, $\varepsilon_{i}=2.53 \times$ $10^{-18} \mathrm{~J}$, and the stagnation pressure and temperature are $0.49 \mathrm{~atm}$ and $9700 \mathrm{~K}$, respectively. Using the Saha equation yields a degree of ionization of $\alpha=0.00623$.

This new estimate for the degree of ionization only changes two values listed in Table 1 , namely, $\alpha=0.00623$ and $n_{e}=1.03 \times$ $10^{19} \mathrm{~m}^{-3}$, which results in a slight modification to the freestream conditions, as seen in Table 2 .

Since the changes to the individual species densities are minimal, and the flow is assumed chemically nonreacting, these slight adjustments to the freestream conditions are assumed not to noticeably alter the resulting flowfield. Therefore, the medium grid, discussed previously, was assumed to provide sufficient resolution and was used in the rest of the analysis. The remaining simulations used the adjusted freestream conditions corresponding to $\alpha=0.00623$.

Figure 13 plots the temperature contours for the flow without the magnetic field. As seen in the figure, the peak temperature is $9000 \mathrm{~K}$, which is $150 \mathrm{~K}$ hotter than the solution computed using the freestream conditions corresponding to $\alpha=0.025$, a result of the slight increase in total density over the conditions reported by Kranc et al. [10]. Using the baseline flowfield solutions, the expected range of electrical conductivity for the various models is displayed in Table 3. The results indicate slight discrepancies in estimated electrical conductivities, with the second-order PRS predictions residing between the semiempirical models.

In the experiment of Kranc et al. [10], the applied magnetic field was produced by an electromagnet located inside the hemisphereshaped forebody. The electromagnet was approximately 1 in. $(0.0254 \mathrm{~m})$ long by $1.25 \mathrm{in} .(0.03175 \mathrm{~m})$ in diameter, with a $0.375 \mathrm{in}$. $(0.0095 \mathrm{~m})$ core. The core was composed of vanadium permandur,

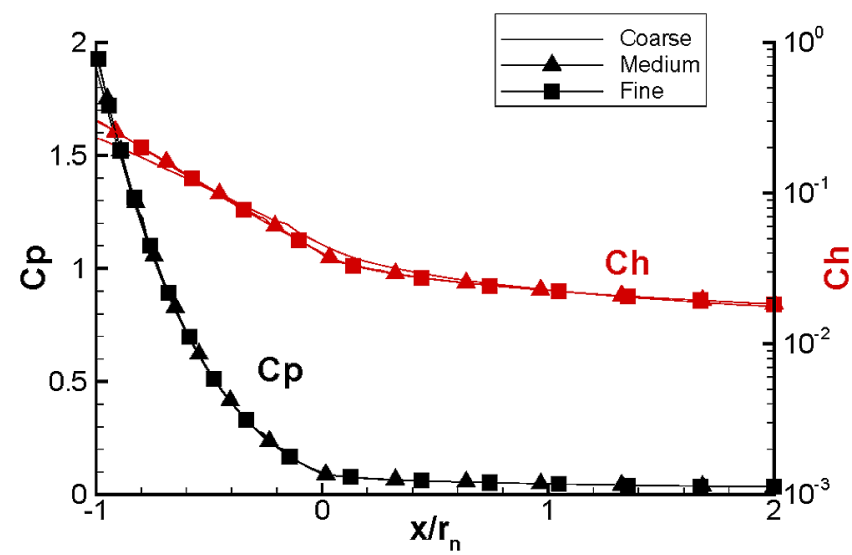

Fig. 12 Nondimensional pressure and heat flux along the surface of Mach 4.75 argon flow around a hemisphere-capped geometry for various grids. 
Table 2 Corrections to the reported freestream conditions for the MHD-heat-shield experiment of Kranc et al. [10]

\begin{tabular}{lcc}
\hline \hline & \multicolumn{2}{c}{ Value } \\
\cline { 2 - 3 } Parameter & Reported & Adjusted \\
\hline$u_{\infty}, \mathrm{m} / \mathrm{s}$ & 3000 & 3000 \\
$T_{\infty}, \mathrm{K}$ & 1100 & 1100 \\
$T_{w}, \mathrm{~K}$ & 300 & 300 \\
$\alpha$ & 0.025 & 0.00623 \\
$\rho_{\mathrm{Ar}}, \mathrm{kg} / \mathrm{m}^{3}$ & $1.01 \times 10^{-4}$ & $1.09 \times 10^{-4}$ \\
$\rho_{\mathrm{Ar}}, \mathrm{kg} / \mathrm{m}^{3}$ & $2.65 \times 10^{-6}$ & $6.85 \times 10^{-7}$ \\
$\rho_{e}, \mathrm{~kg} / \mathrm{m}^{3}$ & $3.64 \times 10^{-11}$ & $9.41 \times 10^{-12}$ \\
\hline
\end{tabular}

and the windings were made of No. 19 Anaconda polyimide enamel (HML)-coated wire with a magnetic resistance of $0.5 \Omega$ [10]. Measurements made by Kranc et al. found the magnet behaved like an ideal dipole, and it is modeled as such. The magnetic field decays as $\mathrm{r}^{-3}$ from its centroid, which is assumed to be located along the $x$ axis, where the forebody merges with the rest of geometry $\left(x / r_{\mathrm{n}}=0\right)$, as seen in Fig. 14. The magnetic moment is aligned along the $x$ axis and is positioned to oppose the incoming flow along the stagnation line. The magnetic field contours are nondimensionalized by the peak magnetic field strength $B_{\max }$, which occurs at the stagnation point $\left(x / \mathrm{r}_{\mathrm{n}}=-1\right.$ for the configuration shown in Fig. 14). Note that the peak magnetic field strength is used to designate each simulation for the rest of this analysis.

In Cartesian coordinates, the ideal dipole magnetic field is

$$
\mathbf{B}=\frac{-B_{\max }}{2\left(x^{2}+y^{2}+z^{2}\right)^{5 / 2}}\left[\begin{array}{c}
2 x^{2}-\left(y^{2}+z^{2}\right) \\
3 x y \\
3 x z
\end{array}\right]
$$

The negative sign in front of the peak field strength $B_{\max }$ is due to the direction of the field flux. The centroid of the dipole is located at the origin.

The flowfield around the geometry is axisymmetric and steady, as evident in the temperature contours seen in Fig. 13. This means the electric current must only travel in the azimuthal direction (perpendicular to the incoming flow, around the axis of symmetry), and the electric field must be zero [4]. This reduces the magnetic force in the momentum equation to $\tilde{\sigma} \cdot(\mathbf{u} \times \mathbf{B}) \times \mathbf{B}$ and sets the energy deposition term in the total energy equation to zero, $\mathbf{j} \cdot \mathbf{E}=0$. Note that joule heating is still present under these assumptions, $(\mathbf{E}+\mathbf{u} \times \mathbf{B}) \cdot \mathbf{j} \neq 0$. Since the electric field is assumed zero and the magnetic field is specified, only the current density field $\mathbf{j}$ needs to be updated in the MHD module.

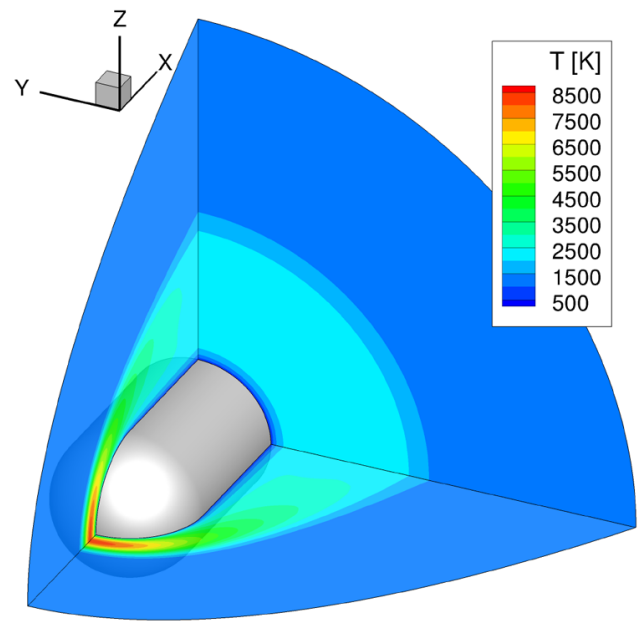

Fig. 13 Temperature contours for Mach 4.75 argon flow around a hemisphere-capped geometry $(\alpha=0.00623)$.
Table 3 Electrical conductivity estimates for the MHD-heat-shield experiment without an applied magnetic fielda

\begin{tabular}{lc}
\hline \hline & $\sigma\left[\Omega^{-1} \mathrm{~cm}^{-1}\right]$ \\
\hline Raizer & $0-1.5$ \\
Chapman and Cowling & $4.4-24.2$ \\
Second-order PRS & $7.1-8.1$ \\
\hline \hline
\end{tabular}

${ }^{\mathrm{a}} \alpha=0.00623$ and $\mathrm{E} / N=0$.

Simulations were carried out at several magnetic field strengths for the different electrical conductivity models. The simulations were started from the steady-state baseline solution (without an applied magnetic field), and iteration was carried until the flowfield achieved a new, converged steady state. Convergence was assumed once the root mean square residual error from the conservation equations decayed to the minimum allowed by machine precision, as seen in Fig. 15 for a typical simulation. In this scenario, at least 10 characteristic flow times worth of time steps are required to achieve a steady-state flowfield solution. A characteristic flow time is defined as the time it takes for the flow to traverse the length of the geometry.

The assumption that the electric field is negligible $(\mathbf{E}=0)$ is verified by simulating the flow with and without a computation of the electric field. The Chapman and Cowling electrical conductivity model was employed for both simulations with $B_{\max }=0.28 \mathrm{~T}$. The MHD module was used to update the electric field every five fluid iterations. Figure 16 plots the temperature contours and current lines

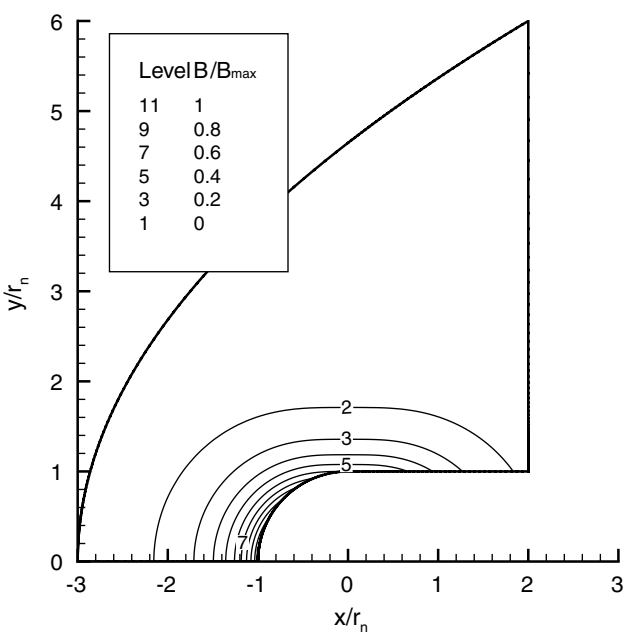

Fig. 14 Nondimensional dipole magnetic field contours from a magnet located in the hemisphere-capped geometry.

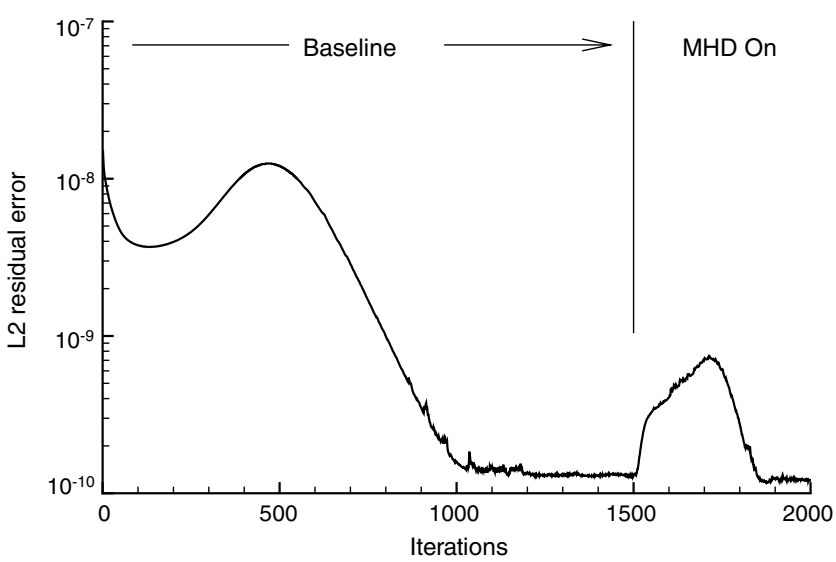

Fig. 15 Root mean square residual error from a simulation of Mach 4.75 argon flow around a hemisphere-capped geometry with a 0.13 T magnet (Chapman and Cowling conductivity model). 


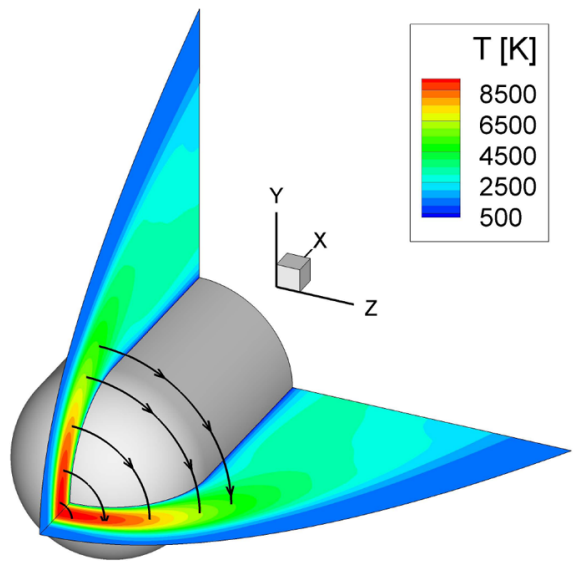

a) Assuming $\mathbf{E}=0$

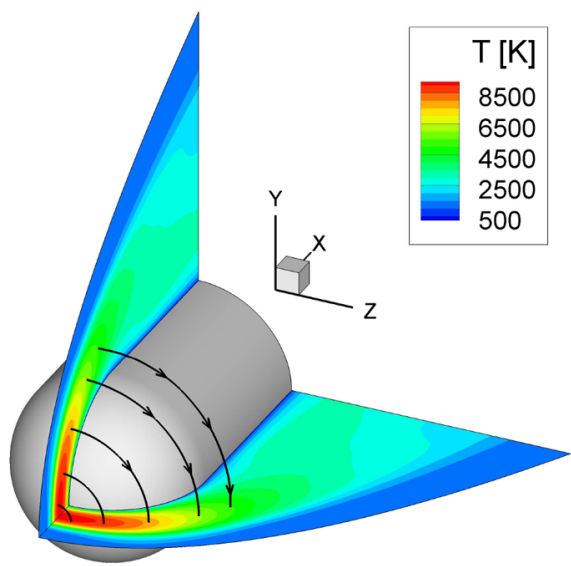

b) Computing $\mathrm{E}$

Fig. 16 Temperature contours and current lines for Mach 4.75 argon flow around a hemisphere-capped geometry with a $0.28 \mathrm{~T}$ magnetic field (Chapman and Cowling conductivity model).

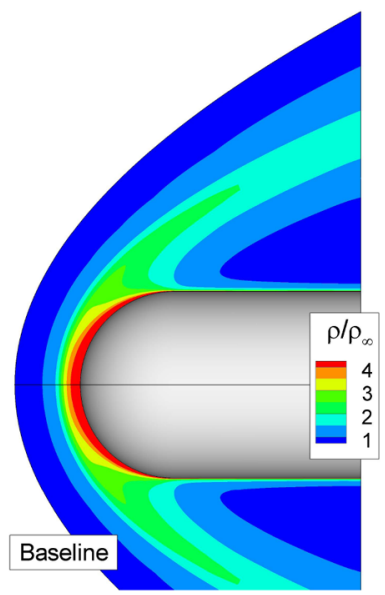

a) Raizer [58]

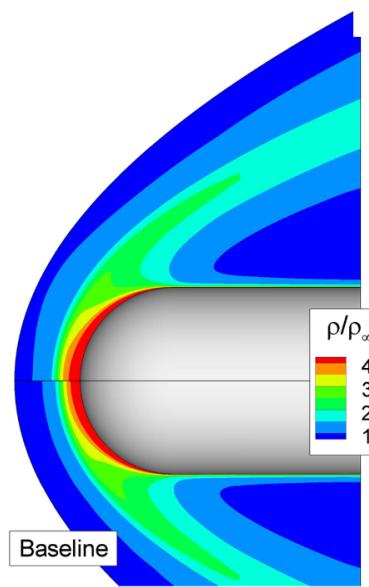

b) Chapman and Cowling

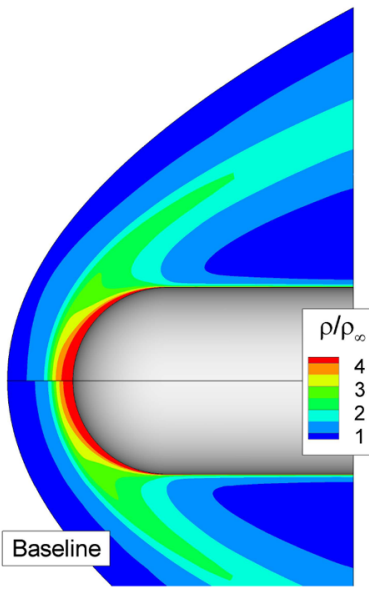

c) $2^{\text {nd }}$ order PRS

Fig. 17 Density ratio contours for Mach 4.75 argon flow around a hemisphere-capped geometry for various electrical conductivity models $\left(B_{\max }=0.13 \mathrm{~T}\right)$.

for both scenarios. As seen in the figures, computing $\mathbf{E}$ from the MHD module does not alter the flow structure or current lines.

Kranc et al. reported an increase in shock standoff distance due to the applied magnetic field [10]. The increase was measured by comparing photographs of the flow with and without the applied magnetic field. In their analysis, they assumed that the upstream edge of the shock can be inferred from the boundary of the flow's luminosity. This photographic technique for measuring the shock standoff distance was previously used by Ziemer [8] and Bailey and Sims [68] in similar experiments.

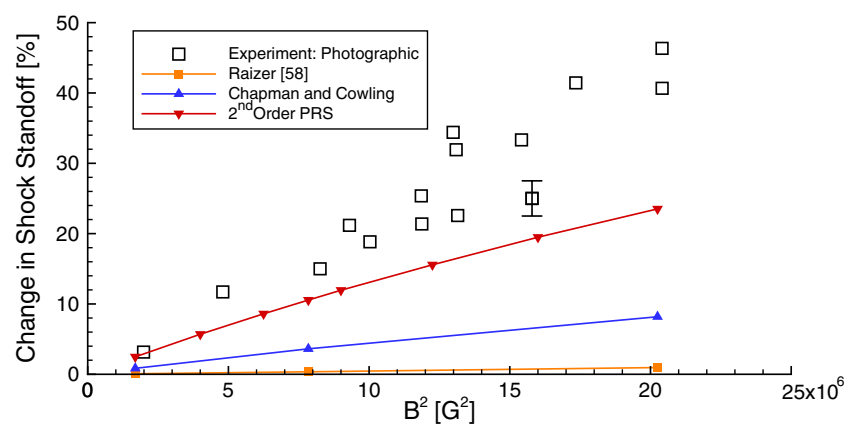

Fig. 18 Percent change in shock standoff distance versus magnetic field strength for Mach 4.75 argon flow around a hemisphere-capped geometry with various electrical conductivity models (measurements from Kranc et al. [10]). (Experimental uncertainty $\pm 10 \%$.)
Although many techniques exist for estimating the shock location, this paper estimates its location to occur where the density ratio exceeds the ideal gas infinite Mach number threshold for a normal shock wave along the stagnation line:

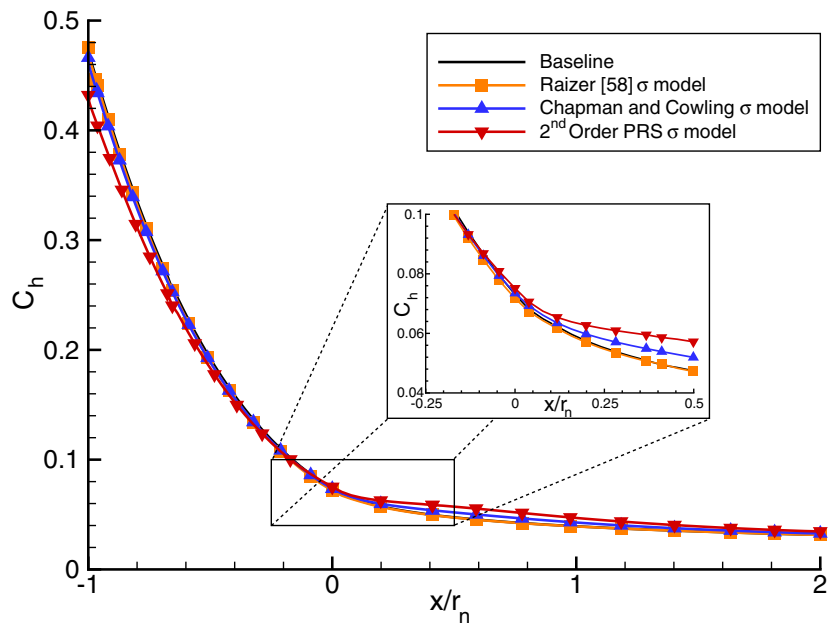

Fig. 19 Nondimensional heat flux along the surface of the geometry for Mach 4.75 argon flow around a hemisphere-capped geometry with a $0.28 \mathrm{~T}$ magnetic field and various electrical conductivity models. 
Table 4 Percent change in heat flux to the surface for Mach 4.75 argon flow around a hemisphere-capped geometry with an MHD heat shield

\begin{tabular}{lcccc}
\hline \hline & \multicolumn{2}{c}{$\Delta$ total heating, \% } & \multicolumn{2}{c}{$\Delta$ peak heating, \% } \\
\cline { 2 - 5 } Model & $B=0.13 \mathrm{~T}$ & $B=0.28 \mathrm{~T}$ & $B=0.13 \mathrm{~T}$ & $B=0.28 \mathrm{~T}$ \\
\hline Raizer & -0.1 & -0.6 & -0.1 & -0.6 \\
Chapman and & 0.3 & 2.2 & -0.7 & -2.5 \\
$\quad$ Cowling & & & -2.7 & -9.7 \\
Second-order PRS & 1.0 & 3.4 & -2.7 \\
\hline \hline
\end{tabular}

$$
\lim _{M_{1} \rightarrow \infty} \frac{\rho_{2}}{\rho_{1}}=\frac{\gamma+1}{\gamma-1}
$$

where $M_{1}$ is the upstream Mach number, $\gamma$ is the ratio of specific heats, $\rho_{1}$ is the freestream density, and $\rho_{2}$ is the downstream density. Using this equation, the density ratio limit for argon is four $(\gamma=5 / 3)$. Figure 17 plots the density ratio contours for the electrical conductivity models with a peak magnetic field of $0.13 \mathrm{~T}$ $\left(1 \mathrm{~T}=10^{4} \mathrm{G}\right)$.

Figure 18 plots the change in shock standoff distance for the simulations and experimental measurements. The experimental measurements were collected using the photographic technique described previously.

The experimental uncertainty (error in determining shock location for one run) is only $\pm 10 \%$, but the repeatability (difference in shock location between nominally identical runs) is $\pm 20 \%$, as seen for $B_{\max }^{2} \simeq 13 \times 10^{6} \mathrm{G}^{2}$. Both the second-order PRS model and the Chapman and Cowling model match the experimental data well at lower magnetic field strengths, but the second-order PRS model better correlates to the experimental measurements at higher magnetic field strengths.

Solutions obtained by using Raizer's [58] electrical conductivity model show almost no change in shock standoff distance, because the model is only dependent on temperature and could not account for the preionized frozen state of the freestream gas. Since the peak temperature occurs just downstream of the bow shock, the model's peak conductivity is also there. However, the magnetic field strength near the shock front is significantly decayed due to its $\mathrm{r}^{-3}$ dependency, which results in only minor changes to the flowfield.

The heat transfer to the surface for the various electrical conductivity models is shown in Fig. 19. The total heating to the geometry is determined by integrating the heat flux over the surface. The change in peak heating is computed by comparing the heat flux at the stagnation point $\left(\Delta q_{w}=\left(q_{w, \mathrm{MHD}}-q_{w \text {, baseline }}\right) / q_{w \text {, baseline }}\right)$. Table 4 lists the percent change in peak heat flux and total heating for various magnetic field strengths and electrical conductivity models.

The total heating to the surface slightly increases because of increased heating to the cylindrical portion of the geometry (i.e., aft of the stagnation region). This is due to the direction of the magnetic field lines in the region where the forebody merges with the cylinder. Results obtained using Raizer's [58] conductivity model fail to capture this behavior (i.e., increased total heating), because its conductivity is not high enough in the aft region, where the direction of the field lines would increase the temperature. In general, an applied magnetic field moderately increases the total heating to the geometry, but it significantly decreases the peak heat flux at the stagnation point. Both the second-order PRS model and the Chapman and Cowling model observe this behavior but, since the second-order PRS model solutions have better agreement with the experimental results, its results for the heat flux to the geometry may be more accurate.

\section{Conclusions}

Newly developed computational tools were used to compute hypersonic flow around a hemisphere-cylinder geometry that uses a magnet located within the body as a means of heat flux mitigation. These tools include an improved electrical conductivity model and a parallelized 3-D MHD module that are loosely coupled to a fluid code. In addition, the Hall effect was implemented and verified by investigating flow between finite electrodes.

Mach 4.75 argon flow over a hemisphere cylinder corresponding to the experiment conducted by Kranc et al. [10] was investigated computationally, using modern CFD techniques. The magnetic field generated from inside the forebody of the geometry worked to oppose and slow the flow near the stagnation region, thus increasing the shock standoff distance. The increase in shock standoff distance decreased the peak heating to the body (at the stagnation point), but it also increased the total heating to the geometry because of increased heating to the aft portion of the body. The changes in shock standoff distance and, consequently, surface heating are dependent on the electrical conductivity model implemented. For this work, a new developed conductivity model based on a PRS to solutions to Boltzmann's equation provided results that gave the closest agreement to experimental measurements. While this work demonstrates the importance the electrical conductivity model has on computing MHD effects for preionized hypersonic flow around a hemisphere cylinder, several other factors, including uncertainty in the reported freestream conditions, surface conditions, the physical size and divergence of the magnet, the Hall effect, and ion slip should also be evaluated to determine if these influence the solution.

These results have important implications for the design of MHDheat-shield devices: they can reduce peak heat loads but with a potential penalty in total heating. Since both peak and total heat loads are important aspects to consider when designing a thermal protection system, this technology provides additional scenarios for vehicle designers to evaluate.

\section{Acknowledgments}

The authors are indebted to the Michigan/U.S. Air Force Research Laboratory/Boeing Collaborative Center in Aeronautical Sciences, which provides funding to the first author. The first author would like to thank Mark Kushner for his valuable discussions on electrical conductivity and the use of his Boltzmann solver.

\section{References}

[1] Kantrowitz, A. R., "A Survey of Physical Phenomena Occurring in Flight at Extreme Speeds," Proceedings of the Conference on HighSpeed Aeronautics, edited by A. Ferri, N. J. Hoff, and P. A. Libby, Polytechnic Inst. of Brooklyn, New York, 1955, pp. 335-339.

[2] Resler, E. L., and Sears, W. R., "The Prospects for MagnetoAerodynamics," Journal of the Aerospace Sciences, Vol. 25, No. 4, April 1958, pp. 235-245, 258.

[3] Resler, E. L., and Sears, W. R., "The Prospects for MagnetoAerodynamics: Correction and Addition," Journal of the Aerospace Sciences, Vol. 26, No. 5, May 1959, p. 318.

[4] Bush, W. B., "Magnetohydrodynamic-Hypersonic Flow Past a Blunt Body," Journal of the Aerospace Sciences, Vol. 25, No. 11, Nov. 1958, pp. 685-690.

[5] Bush, W. B., "The Stagnation-Point Boundary Layer in the Presence of an Applied Magnetic Field," Journal of the Aerospace Sciences, Vol. 28, No. 8, Aug. 1961, pp. 610-611.

[6] Poggie, J., and Gaitonde, D. V., "Magnetic Control of Flow Past a Blunt Body: Numerical Validation and Exploration," Physics of Fluids, Vol. 14, No. 5, May 2002, pp. 1720-1731. doi:10.1063/1.1465424

[7] Coakley, J. F., and Porter, R. W., "Time-Dependent Numerical Analysis of MHD Blunt Body Problem," AIAA Journal, Vol. 9, No. 8, Aug. 1971, pp. $1624-1626$. doi: $10.2514 / 3.49966$

[8] Ziemer, R. W., "Experimental Investigations in Magnetoaerodynamics," ARS Journal, Vol. 29, No. 9, Sept. 1959, p. 642.

[9] Wilkinson, J. B., "Magnetohydrodynamic Effects on Stagnation-Point Heat Transfer from Partially Ionized Nonequilibrium Gases in Supersonic Flow," Proceedings of the 3rd Symposium of Engineering Aspects of Magnetohydrodynamics, edited by N. W. Mather, and G. W. Sutton, Gordon and Breach, New York, 1964, pp. 413-438.

[10] Kranc, S., Yuen, M. C., and Cambel, A. B., "Experimental Investigation of Magnetoaerodynamic Flow Around Blunt Bodies," NASA, CR 1393, Aug. 1969.

[11] Nowak, R. J., Kran, S., Porter, R. W., Yuen, M. C., and Cambel, A. B., "Magnetogasdynamic Re-Entry Phenomena," Journal of Spacecraft 
and Rockets, Vol. 4, No. 11, Nov. 1967, pp. 1538-1542. doi: $10.2514 / 3.29125$

[12] Nowak, R. J., and Yuen, M. C., "Heat Transfer to a Hemispherical Body in Supersonic Argon Plasma," AIAA Journal, Vol. 11, No. 11, Nov. 1973, pp. 1463-1464. doi:10.2514/3.50611

[13] Porter, R. W., and Cambel, A. B., "Hall Effect in Flight Magnetogasdynamics," AIAA Journal, Vol. 5, No. 12, Dec. 1967, pp. 2208-2213.

doi: $10.2514 / 3.4410$

[14] Romig, M. F., "The Influence of Electric and Magnetic Fields on Heat Transfer to Electrically Conducting Fluids," Advances in Heat Transfer, edited by T. F. Irvine, and J. P. Hartnett, Vol. 1, Academic, New York, 1964, pp. 267-354.

[15] Padilla, J. F., "Assessment of Gas-Surface Interaction Models for Computation of Rareed Hypersonic Flows," Ph.D. Thesis, Department of Aerospace Engineering, Univ. of Michigan, Ann Arbor, MI, 2008.

[16] Fomin, V. M., Tretyakov, P. K., and Taran, J.-P., "Flow Control using Various Plasma and Aerodynamic Approaches," Aerospace Science and Technology, Vol. 8, No. 5, July 2004, pp. 411-421. doi:10.1016/j.ast.2004.01.005

[17] Shang, J. S., Surzhikov, S. T., Kimmel, R., Gaitonde, D., Menart, J., and Hayes, J., "Mechanisms of Plasma Actuators for Hypersonic Flow Control," Progress in Aerospace Sciences, Vol. 41, No. 8, Nov. 2005, pp. $642-668$ doi:10.1016/j.paerosci.2005.11.001

[18] Bityurin, V., Bocharov, A., and Lineberry, J., "MHD Flow Control in Hypersonic Flight," AIAA Paper 2005-3225, May 2005.

[19] Palmer, G., "Magnetic Field Effects on the Computed Flow over a Mars Return Aerobrake," Journal of Thermophysics and Heat Transfer, Vol. 7, No. 2, April-June 1993, pp. 294-301. doi: $10.2514 / 3.419$

[20] Bisek, N. J., Boyd, I. D., and Poggie, J., "Numerical Study of PlasmaAssisted Aerodynamic Control for Hypersonic Vehicles," Journal of Spacecraft and Rockets, Vol. 46, No. 3, May-June 2009. doi: $10.2514 / 1.39032$

[21] Kremeyer, K., Sebastian, K., and Shu, C.-W., "Computational Study of Shock Mitigation and Drag Reduction by Pulsed Energy Lines," AIAA Journal, Vol. 44, No. 8, Aug. 2006, pp. 1720-1731. doi: $10.2514 / 1.17854$

[22] Yan, H., and Gaitonde, D., "Control of Edney IV Interaction by Energy Pulse," AIAA Paper 2006-562, Jan. 2006.

[23] Menart, J., Staneld, S., Shang, J., Kimmel, R., and Hayes, J., "Study of Plasma Electrode Arrangements for Optimum Lift in a Mach 5 Flow," AIAA Paper 2006-1172, Jan. 2006.

[24] Girgis, I. G., Shneider, M. N., Macheret, S. O., Brown, G. L., and Miles, R. B., "Creation of Steering Moments in Supersonic Flow by Off-Axis Plasma Heat Addition," AIAA Paper 2002-129, Jan. 2002.

[25] Gnemmi, P., Charon, R., Duperoux, J.-P., and George, A., "Feasibility Study for Steering a Supersonic Projectile by a Plasma Actuator," AIAA Journal, Vol. 46, No. 6, June 2008, pp. 1308-1317. doi: $10.2514 / 1.24696$

[26] Bityurin, V. A., Vatazhin, A. B., and Gus'kov, O. V., "Hypersonic Flow Past the Spherical Nose of a Body in the Presence of a Magnetic Field," Fluid Dynamics, Vol. 39, No. 4, July 2004, pp. 657-666. doi:10.1023/B:FLUI.0000045682.98147.db

[27] Miles, R. B., Macheret, S. O., Shneider, M. N., Steeves, C., Murray, R. C., Smith, T., and Zaidi, S. H., "Plasma-Enhanced Hypersonic Performance Enabled by MHD Power Extraction," AIAA Paper 2005561, Jan. 2005.

[28] Fujino, T., Matsumoto, Y., Kasahara, J., and Ishikawa, M., "Numerical Studies of Magnetohydro-dynamic Flow Control Considering Real Wall Electrical Conductivity," Journal of Spacecraft and Rockets, Vol. 44, No. 3, May-June 2007, pp. 625-632. doi: $10.2514 / 1.25824$

[29] Katsurayama, H., Kawamura, M., Matsuda, A., and Abe, T., "Kinetic and Continuum Simulations of Electromagnetic Control of a Simulated Reentry Flow," Journal of Spacecraft and Rockets, Vol. 45, No. 2, March-April 2008, pp. 248-254. doi:10.2514/1.31702

[30] Yoshino, T., Fujino, T., and Ishikawa, M., "Possibility of Thermal Protection in Earth Re-Entry Flight by MHD Flow Control with AirCore Circular Magnet," IEEJ Transactions on Electrical and Electronic Engineering, Vol. 4, No. 4, July 2009, pp. 510-517. doi: $10.1002 /$ tee. 20437

[31] Hodara, H., "The Use of Magnetic Fields in the Elimination of the ReEntry Radio Blackout," Proceedings of the IRE, Vol. 49, No. 12, Dec. 1961 , pp. 1825-1830.
doi:10.1109/JRPROC.1961.287709

[32] Kim, M., Keidar, M., and Boyd, I. D., "Analysis of an Electromagnetic Mitigation Scheme for Reentry Telemetry Through Plasma," Journal of Spacecraft and Rockets, Vol. 45, No. 6, Nov.-Dec. 2008, pp. 12231229.

doi:10.2514/1.37395

[33] Macheret, S. O., Shneider, M. N., and Candler, G. V., "Modeling of MHD Power Generation on Board Reentry Vehicles," AIAA Paper 2004-1024, Jan. 2004.

[34] Wan, T., Suzuki, R., Candler, G., Macheret, S., and Schneider, M., "Three Dimensional Simulation of Electric Field and MHD Power Generation During Re-Entry," AIAA Paper 2005-5045, June 2005

[35] Fujino, T., Yoshino, T., and Ishikawa, M., "Prediction of Generator Performance and Aerodynamic Heating of Reentry Vehicle Equipped with On-Board Surface Hall Type MHD Generator," AIAA Paper 20084225, June 2008.

[36] Bityurin, V. A., Bocharov, A. N., and Lineberry, J. T., "Results of Experiments on MHD Hypersonic Flow Control," AIAA Paper 20042263, June 2004.

[37] Takizawa, Y., Sato, S., Abe, T., and Konigorski, D., "Electro-Magnetic Effect on Shock Layer Structure in Reentry-Related High-Enthalpy Flow," AIAA Paper 2004-2162, June 2004

[38] Kimmel, R., Hayes, J., Menart, J., and Shang, J., "Supersonic Plasma Flow Control Experiments," U.S. Air Force Research Laboratory, TR ARFL-VA-WP-TR-2006-3006, Wright-Patterson AFB, OH, Dec. 2005

[39] Matsuda, A., Kawamura, M., Takizawa, Y., Otsu, H., Konigorski, D., Sato, S., and Abe, T., "Experimental Investigation of the Hall Effect for the Interaction between the Weakly-Ionized Plasma Flow and Magnetic Body," AIAA Paper 2007-1437, Jan. 2007.

[40] Gülhan, A., Esser, B., Koch, U., Siebe, F., Riehmer, J., Giordano, D., and Konigorski, D., "Experimental Verication of Heat-Flux Mitigation by Electromagnetic Fields in Partially-Ionized-Argon Flows," Journal of Spacecraft and Rockets, Vol. 46, No. 2, March-April 2009, pp. 274 283. doi: $10.2514 / 1.39256$

[41] Gaitonde, D. V., and Poggie, J., "An Implicit Technique for 3-D Turbulent MGD with the Generalized Ohm's Law," AIAA Paper 20012736, June 2001.

[42] Gaitonde, D. V., "A High-Order Implicit Procedure for the 3-D Electric Field in Complex Magnetogasdynamic Simulations," Computers and Fluids, Vol. 33, No. 3, March 2004, pp. 345-374. doi:10.1016/j.compfluid.2003.06.001

[43] Gaitonde, D. V., and Poggie, J., "An Implicit Technique for ThreeDimensional Turbulent Magnetoaerodynamics," AIAA Journal, Vol. 41, No. 11, Nov. 2003, pp. 2179-2191. doi: $10.2514 / 2.6810$

[44] Damevin, H.-M., and Hoffmann, K. A., "Numerical Simulations of Magnetic Flow Control in Hypersonic Chemically Reacting Flows," Journal of Thermophysics and Heat Transfer, Vol. 16, No. 4, Oct.Dec. 2002, pp. 498-507. doi: $10.2514 / 2.6724$

[45] Scalabrin, L. C., and Boyd, I. D., "Development of an Unstructured Navier-Stokes Solver For Hypersonic Nonequilibrium Aerothermodynamics," AIAA Paper 2005-5203, June 2005.

[46] Scalabrin, L. C., and Boyd, I. D., "Numerical Simulation of Weakly Ionized Hypersonic Flow for Reentry Configurations," AIAA Paper 2006-3773, June 2006.

[47] MacCormack, R. W., and Candler, G. V., "The solution of the NavierStokes Equations Using Gauss-Seidel Line Relaxation," Computers and Fluids, Vol. 17, No. 1, Jan. 1989, pp. 135-150. doi:10.1016/0045-7930(89)90012-1

[48] Holman, T. D., and Boyd, I. D., "Numerical Investigation of the Effects of Continuum Breakdown on Hypersonic Vehicle Surface Properties," AIAA Paper 2008-3928, June 2008.

[49] Shercli, J., A Textbook of Magnetohydrodynamics, Pergamon, New York, 1965

[50] Gaitonde, D. V., and Poggie, J., "Elements of a Numerical Procedure for 3-D MGD Flow Control Analysis," AIAA Paper 2002-198, Jan. 2002.

[51] Bisek, N. J., Boyd, I. D., and Poggie, J., "Numerical Study of Electromagnetic Aerodynamic Control of Hypersonic Vehicles," AIAA Paper 2009-1000, Jan. 2009.

[52] Bisek, N. J., Boyd, I. D., and Poggie, J., "Three Dimensional Simulations of Hypersonic MHD Flow Control," AIAA Paper 20093731, Jan. 2009.

[53] Hurwitz, H., Jr., Kilb, R. W., and Sutton, G. W., "Influence of Tensor Conductivity on Current Distribution in MHD Generator," Journal of Applied Physics, Vol. 32, No. 2, Feb. 1961, pp. 205-216. doi:10.1063/1.1735979 
[54] Oliver, D. A., and Mitchner, M., "Nonuniform Electrical Conduction in MHD Channel," AIAA Journal, Vol. 5, No. 8, Aug. 1967, pp. 14241432. doi: $10.2514 / 3.4215$

[55] White, F. M., Viscous Fluid Flow, 3rd ed., McGraw-Hill, New York, 2006.

[56] Sutton, G. W., and Sherman, A., Engineering Magnetohydrodynamics, McGraw-Hill, New York, 1965.

[57] Lin, S.-C., Resler, E. L., and Kantrowitz, A., "Electrical Conductivity of Highly Ionized Argon Produced by Shock Waves," Journal of Applied Physics, Vol. 26, No. 1, Jan. 1955, pp. 95-109. doi: $10.1063 / 1.1721870$

[58] Raizer, Y. P., Gas Discharge Physics, Springer-Verlag, New York1991.

[59] Cambel, A. B., Plasma Physics and Magnetouid-Mechanics, McGrawHill, New York, 1963.

[60] Bird, G. A., Molecular Gas Dynamics and the Direct Simulation of Gas Flows, Oxford Univ. Press, Oxford, England, U.K., 1994.

[61] Bird, G. A., "Monte-Carlo Simulation in an Engineering Context," Progress of Astronautics and Aeronautics: Rarefied Gas Dynamics, edited by S. S. Fisher, Vol. 74, AIAA, New York, 1981, pp. 239-255.

[62] Weng, Y., and Kushner, M. J., "Method for Including Electron-Electron Collisions in Monte Carlo Simulations of Electron Swarms in Partially Ionized Gases," Physical Review A, Vol. 42, No. 10, Nov. 1990, pp. 6192-6200.

doi:10.1103/PhysRevA.42.6192

[63] Viana, F. A. C., and Goel, T., "SURROGATES Toolbox User's Guide," Univ. of Florida, Gainesville, FL, 2008.

[64] Makridakis, S., "Accuracy Measures: Theoretical and Practical Concerns," International Journal of Forecasting, Vol. 9, No. 4, Dec. 1993 , pp. $527-529$. doi:10.1016/0169-2070(93)90079-3

[65] Schwartzentruber, T. E., Scalabrin, L. C., and Boyd, I. D., "Hybrid Particle-Coontinuum Simulations of Non-Equilibrium Hypersonic Blunt Body Flow Fields," AIAA Paper 2006-3602, June 2006.

[66] Arave, R. J., and Huseley, O. A., "Aerothermodynamic Properties of High Temperature Argon," Boeing, TR D2-11238, Seattle, WA, Feb. 1962.

[67] Messerle, H. K., Magnetohydrodynamic Electrical Power Generation, Wiley, New York, 1995.

[68] Bailey, A. B., and Sims, W. H., "Shock Detachment Distance for Blunt Bodies in Argon at Low Reynolds Number," AIAA Journal, Vol. 1, No. 12 , Dec. 1963 , pp. $2867-2868$. doi: $10.2514 / 3.2195$

A. Ketsdever Associate Editor 